\title{
Short communication: Effects of oral flavonoid supplementation on the metabolic and antioxidative status of newborn dairy calves
}

\author{
J. Maciej, ${ }^{*}$ C. T. Schäff, ${ }^{*}$ E. Kanitz,† A. Tuchscherer,‡ R. M. Bruckmaier,§ S. Wolffram,\# and H. M. Hammon* \\ *Institute of Nutritional Physiology "Oskar Kellner," \\ †Institute of Behavioural Physiology, and \\ łInstitute of Genetics and Biometry, Leibniz Institute for Farm Animal Biology (FBN), 18196 Dummerstorf, Germany \\ $\S$ Department of Clinical Research and Veterinary Public Health, Veterinary Physiology, Vetsuisse Faculty, University of Bern, 3001 Bern, \\ Switzerland \\ \#Institute of Animal Nutrition and Physiology, Christian Albrechts University Kiel, 24118 Kiel, Germany
}

\begin{abstract}
Scientific proof for flavonoids as a health tool in calf nutrition is inconsistent. We investigated the effects of the most abundant flavonoid, quercetin, and of a green tea extract (GTE) containing various catechins on the metabolic and antioxidative traits in dairy calves to clarify their potential health-promoting effects. Male newborn German Holstein calves ( $\mathrm{n}=7$ per group) received either no flavonoid (control group), $10 \mathrm{mg}$ of quercetin equivalents as quercetin aglycone or as rutin/ $\mathrm{kg}$ of body weight (BW) per day, or $10 \mathrm{mg} / \mathrm{kg}$ of BW per day of a GTE from d 2 to 26 of life. The supplements were provided with the morning and evening feeding. The calves were fed colostrum and milk replacer, and BW, feed intake, and health status were evaluated daily. Blood samples were collected from a jugular vein on d 1, 5, 12, 19, and 26 before the morning feeding to investigate the metabolic and antioxidative status of the calves. The growth performance and health status remained unchanged, but the GTE-fed calves had fewer loose feces than the controls. The plasma concentrations of quercetin changed over time and were higher in the rutin-fed group than in the control group, whereas the catechins were below the detection limit. The plasma Trolox equivalent antioxidative capacity and ferric reducing ability of plasma were measured as markers for plasma antioxidative capacity. The concentrations of Trolox equivalent antioxidative capacity increased, whereas ferric reducing ability of plasma decreased after the first day of life in all the groups. The oxidative stress markers in the plasma were measured as thiobarbituric acid reactive substances and F2-isoprostanes, but these did not indicate treatment or time effects. The plasma concentrations of total protein, albumin, urea, lactate, glucose, and nonesterified fatty acids and of insulin and
\end{abstract}

Received June 3, 2015.

Accepted September 24, 2015.

${ }^{1}$ Corresponding author: hammon@fbn-dummerstorf.de cortisol varied over time, but no group differences were caused by the flavonoid supplementation. In summary, orally administered quercetin and catechins at the dosages used in the present study resulted in weak effects on health and no effects on the metabolic and antioxidative status of newborn dairy calves.

Key words: antioxidative status, calf, flavonoid, quercetin, rutin

\section{Short Communication}

Calf losses mainly occur during the first week of life, mostly because of respiratory and digestive problems (USDA, 2011). Good management, such as an early and sufficient colostrum supply, is essential for optimal postnatal development. In addition, feed supplements are a daily practice of modern dairy farms. Particularly since the ban of antibiotic growth promoters in the European Union in 2006, the call for "natural" feed additives has gained enormous popularity. In this respect, flavonoids, as secondary plant metabolites, ubiquitous in all higher plants, are of interest. Their health-promoting properties are mainly thought to be due to their strong antioxidant activity shown in vitro, associated with effects on inflammatory cells (Middleton et al., 2000), glucose and lipid metabolism (Shetty et al., 2004; Kobayashi et al., 2010), or incidence of diarrhea (di Carlo et al., 1994). One of the most abundant flavonoids is the flavonol quercetin, a pentahydroxyflavon, mostly bound in a $\beta$-glycosidic manner to at least one sugar molecule and present in high concentrations in apples and onions. Quercetin bound to rutinose is called rutin and is the major glycoside of quercetin. The predominant flavanol, another subgroup of flavonoids, is catechin, which is mainly found in high concentrations in green tea.

Almost any disease is associated with increased formation of reactive oxygen species, thus causing oxidative stress (Halliwell, 1991). In calves, enhanced oxidative stress has been seen on the first day of life (Alex- 
androvich and Antonovna, 2009), and especially if the calf was sick (Ahmed and Hassan, 2007). Because the colostrum supply for newborn calves is often impaired, this possibly affects the antioxidant system (Blum et al., 1997). Thus, improvement of the antioxidant status in newborn calves may accelerate the maturation of their own immune system, improve health status, and thus reduce calf losses. In a previous study, we have shown that quercetin in newborn calves is bio-available when fed as quercetin aglycone or as rutin, a prerequest for systemic functioning of quercetin in the body (Maciej et al., 2015). Based on that, we have tested in the present study the hypothesis that quercetin and catechin supplementation affect metabolism and the antioxidative and health status during the first 4 wk of life in dairy calves.

The procedures in this study were performed in accordance with the German animal protection law and approved by the relevant authorities (Landesamt für Landwirtschaft, Lebensmittelsicherheit und Fischerei, Mecklenburg-Vorpommern, Germany). Twenty-eight male German Holstein calves were examined from d 1 to 26 of life. All the calves were spontaneously born from multiparous cows on neighboring farms and transported directly after birth to the experimental barn of the University of Rostock in Dummerstorf, where they were kept in single boxes with straw bedding. The calves had free access to water and were fed twice daily by a nipple bottle or nipple bucket. For the first $3 \mathrm{~d}$ of life, the calves received pooled colostrum obtained from milkings 1 , 3, and 5 (d 1, 2, and 3 after parturition, respectively) in amounts of $8 \%$ of $\mathrm{BW}$ on $\mathrm{d} 1$ and $10 \%$ of BW on d 2 and 3 (Supplemental Table S1; http:// dx.doi.org/10.3168/jds.2015-9906). From d 4 until d 26, the calves received a commercial milk replacer (150 g/L; SalvaLac MiraPro 45, Salvana Tiernahrung GmbH, Klein-Offenseth Sparrieshoop, Germany) in the amount of $12 \%$ of $\mathrm{BW} / \mathrm{d}$. To ensure equal feed uptake in all the groups, the refused amounts of colostrum or milk replacer were tube fed. The milk replacer amounts were adapted to BW once per week.

From d 2 to 6 , colostrum or milk replacer was supplemented with chicken-egg-derived immunoglobulins (Globigen Life Start 25\%, EW Nutrition GmbH, Visbek, Germany) fed twice daily in amounts of 40,32, 24, 16, and $8 \mathrm{~g} / \mathrm{d}$, respectively (Maciej et al., 2015). The calves had free access to pelleted concentrate (Kälber Start 18/3 Vollkraft Mischfutterwerke GmbH, Karstädt, Germany) and hay from d 4 on. Concentrate intake was measured daily after the morning milk feeding (Maciej et al., 2015).

The health status of the calves was determined daily by measuring the rectal temperature, heart and respiratory rate, nasal discharge, respiratory sounds, and by navel inspection. The fecal consistency was assessed daily by fecal consistency score according to Larson et al. (1977): normal (1), soft (2), runny (3), or watery (4). Sick calves were treated by a veterinarian accordingly.

The calves were randomly assigned to 1 of 4 feeding groups $(\mathrm{n}=7)$. Control (CTRL) received no flavonoids, QA received $10 \mathrm{mg} /(\mathrm{kg}$ of $\mathrm{BW} \times \mathrm{d})$ quercetin aglycone (quercetin dihydrate; Carl Roth $\mathrm{GmbH} \& \mathrm{Co}$. KG, Karlsruhe, Germany), RU received $20 \mathrm{mg} /(\mathrm{kg}$ of $\mathrm{BW} \times \mathrm{d}$ ) of quercetin as glucorhamnoside rutin (rutin trihydrate; Carl Roth $\mathrm{GmbH} \&$ Co. KG), and CA received $10 \mathrm{mg} /(\mathrm{kg}$ of $\mathrm{BW} \times \mathrm{d})$ of a green tea extract (GTE) containing various catechins (Polyphenon 60; Sigma-Aldrich Chemie GmbH, Steinheim, Germany). For QA and RU, the daily dose of quercetin equivalents was $10 \mathrm{mg} / \mathrm{kg}$ of BW $(30 \mu \mathrm{mol} / \mathrm{kg}$ of BW $)$. The GTE (70.3\% total catechins) fed in CA was composed of $1.4 \%$ catechin, $0.3 \%$ catechin gallate, $5.2 \%$ gallocatechin, and $2.1 \%$ gallocatechin gallate (all as trans-isomers) and $6.4 \%$ epicatechin, $7.0 \%$ epicatechin gallate, $19.0 \%$ epigallocatechin, and $28.8 \%$ epigallocatechin gallate (all as cis-isomers). The amounts of flavonoid fed to the calves in this study were based on previous studies in pigs (Lesser et al., 2004; Lühring et al., 2011). Due to studies on the bioavailability of flavonoids, all calves received their respective daily dose with the morning feeding on d 2, and no flavonoids were fed on $\mathrm{d} 3$ and 4 (Maciej et al., 2015). From d 5 on, the daily dose was equally split between the morning and evening meal until d 26. The flavonoids were suspended in water and administered with a disposable $10-\mathrm{mL}$ syringe directly into the mouth during milk feeding.

Jugular blood samples were taken immediately after birth, and on d 5, 12, 19, and 26 before morning feeding using evacuated tubes (Vacuette, Greiner Bio-One GmbH, Frickenhausen, Germany). Tubes containing 15 $\mathrm{IU} / \mathrm{mL}$ of lithium heparinate were used for the determination of the plasma concentrations of flavonols, catechins, and the markers for antioxidative capacity and oxidative stress; tubes containing $2.5 \mathrm{~g} / \mathrm{L}$ of sodium fluoride and $1.8 \mathrm{~g} / \mathrm{L}$ potassium EDTA were used for the determination of plasma protein, albumin, glucose, NEFA, urea, and lactate, and tubes containing $1.8 \mathrm{~g} / \mathrm{L}$ of potassium-EDTA were used for the determination of plasma insulin and cortisol concentrations. The blood samples were immediately put on ice and centrifuged $\left(1,500 \times g, 4^{\circ} \mathrm{C}, 20 \mathrm{~min}\right)$. To measure the catechins, 1 $\mathrm{mL}$ of plasma was mixed with $20 \mu \mathrm{L}$ of an ascorbateEDTA solution $\left(0.4 \mathrm{~mol} / \mathrm{L}\right.$ of $\mathrm{NaH}_{2} \mathrm{PO}_{4}$; Carl Roth GmbH \& Co. KG) containing 20\% ascorbic acid (Merck KGaA, Darmstadt, Germany) and 0.1\% EDTA (Carl Roth GmbH \& Co. KG) at pH 3.6. To measure the F2isoprostanes, a $50 \mu \mathrm{g} / \mathrm{mL}$ ethanolic butylhydroxytoluene solution $(50 \mu \mathrm{g} / \mathrm{mL}$ of ethanol, wt/vol) was added 
in $1 \%$ proportion for stabilization. The plasma samples were stored until analyzed at $-20^{\circ} \mathrm{C}$ for metabolites and hormones and at $-80^{\circ} \mathrm{C}$ for flavonoid and (anti) oxidative parameters.

The plasma concentrations of quercetin aglycone and its methylated (isorhamnetin and tamarixetin) and dehydroxylated (kaempferol) derivatives were analyzed by HPLC with fluorescence detection as previously described (Berger et al., 2012). The detection limit of the flavonol was $\leq 10 \mathrm{nmol} / \mathrm{L}$, and the recovery rate of the flavonols was $92 \pm 2 \%$ (mean \pm SEM). The inter- and intraassay coefficients of variability for the quercetin were 7.2 and $0.5 \%$, respectively. The sum of quercetin and its metabolites in the plasma is referred to as total flavonols.

The plasma concentrations of the individual catechins (gallocatechin, epigallocatechin, epigallocatechin gallate, epicatechin gallate, and epicatechin) were determined by HPLC (Lee et al., 1995) with some modifications (Egert et al., 2013). Quantification of the individual plasma catechins was carried out using external standards, which were generated by simultaneously adding catechin, gallocatechin, epigallocatechin, epigallocatechin gallate, epicatechin gallate, and epicatechin (Carl Roth GmbH \& Co. KG) to untreated plasma. The calibration samples were treated in the same way as the experimental samples. The coefficient of determination was $\mathrm{r} \geq 0.99$, the detection limit of the catechins was $\leq 10 \mathrm{nmol} / \mathrm{L}$, and the intraassay coefficient of variation was $2.4 \pm 2.6$ to $6.5 \pm 4.7 \%$.

The Trolox equivalent antioxidative capacity (TEAC) is defined as the amount of the water-soluble vitamin $\mathrm{E}$ derivative Trolox (in $\mathrm{mmol}$ ) needed to show the same antioxidant capacity at a defined time point as $1 \mathrm{mmol}$ of plasma. It is expressed as Trolox equivalents (TE) in millimoles per liter of plasma. The TEAC was measured according to the modified protocol of Re et al.. (1999) by measuring spectrophotometrically at a wavelength of $734 \mathrm{~nm}$ the decolorization of the 2,2'-azinobis(3-ethylbenzothiazoline-6-sulfonic acid) radical cation $\left(\mathrm{ABTS}^{+}\right)$caused by binding to antioxidants. The ferric reducing ability of plasma (FRAP) was determined according to Benzie and Strain (1996) and is given in ascorbic acid equivalents in micromoles per liter of plasma. The reduction of $\mathrm{Fe}^{3+}$-tripyridyltriazin by antioxidants from plasma results in a color change that is directly proportional to the antioxidative capacity. The absorbance was measured at a wavelength of $595 \mathrm{~nm}$ and compared with the absorbance of ascorbic acid. The thiobarbituric acid reactive substance concentration in plasma was measured according to Yagi (1998) with modifications and is given as malondialdehyde equivalents in micromoles per liter of plasma. Adding thiobarbituric acid to the sample results in the formation of a red complex, whose optical density was measured photometrically at a wavelength of $532 \mathrm{~nm}$. The F2-isoprostanes, especially their main representative 8-iso-prostaglandin $\mathrm{F}_{2 \alpha}$, are produced in vivo by peroxidation of the cell membrane lipid component arachidonic acid, and they are considered to be a reliable marker for lipid peroxidation. Detection of 8-iso-prostaglandin $\mathrm{F}_{2 \alpha}$ was carried out with an enzymatic immune assay (Direct 8-iso-prostaglandin $\mathrm{F}_{2 \alpha}$; Assay Designs, MI).

The plasma metabolites were analyzed by the Clinic for Cattle (University of Veterinary Medicine Hannover, Foundation, Germany) spectrophotometrically on an ABX Pentra 400 (Horiba ABX SAS, Montpellier Cedex, France) using the respective kits (Maciej et al., 2015). The plasma insulin and cortisol concentrations were measured by RIA or by ELISA, respectively, as previously described (Maciej et al., 2015).

The colostrum was analyzed for DM, CP, crude fat, and ash content were measured by MQD (Qualitätsprüfungs- und Dienstleistungsgesellschaft Mecklenburg-Vorpommern, Güstrow, Germany) using standard procedures according to Weende (Naumann and Basler, 2004). The chemical components and contents of the milk replacer were provided by the manufacturer according to standard procedures (Naumann and Basler, 2004; Supplemental Table S1; http://dx.doi. org/10.3168/jds.2015-9906).

Statistical analyses were performed using SAS software (SAS Institute Inc., Cary, NC). The data on performance and from the plasma measurements are presented as least squares means \pm standard error and were analyzed by repeated measurement ANOVA using the Mixed procedure of SAS/STAT software with a model containing as the fixed effects the treatment group (levels: CTRL, QA, RU, CA; for plasma flavonol concentrations only CTRL, QA, RU), day (levels on $\mathrm{d} 1,5,12,19,26)$, and group $\times$ day interactions. Repeated measures on the same animal were taken into account by the REPEATED statement of the MIXED procedure and an autoregressive type for the block diagonal residual covariance matrix. Pairwise differences among treatment groups and days were tested by the Tukey-Kramer test. The data on fecal score are presented as the mean $\pm \mathrm{SE}$ and were analyzed with the NPAR1WAY procedure of the SAS/STAT software across the one-way classification treatment group (levels: CTRL, QA, RU, CA) using Wilcoxon scores (for each week separately). The effects and differences were considered significant if $P<0.05$.

The plasma concentrations of the total flavonols in $\mathrm{QA}$ and RU increased $(P<0.05)$ until $\mathrm{d} 12$ but decreased thereafter in QA (Figure 1). The plasma flavonol concentrations were relatively low because 
Table 1. Weekly fecal score of calves fed no flavonoid (CTRL), quercetin aglycone (QA), rutin (RU), or a green tea extract containing mainly catechins (CA) from d 5 to $26^{1}$

\begin{tabular}{lllll}
\hline & \multicolumn{3}{c}{ Group } \\
\cline { 2 - 5 } Time & CTRL & RU & QA & CA \\
\hline Week 1 & $1.5 \pm 0.2^{\mathrm{a}}$ & $1.2 \pm 0.1^{\mathrm{ab}}$ & $1.3 \pm 0.1^{\mathrm{ab}}$ & $1.0 \pm 0.01^{\mathrm{b}}$ \\
Week 2 & $1.7 \pm 0.3$ & $1.4 \pm 0.2$ & $1.7 \pm 0.2$ & $1.6 \pm 0.2$ \\
Week 3 & $1.0 \pm 0.2$ & $1.7 \pm 0.4$ & $1.5 \pm 0.2$ & $1.6 \pm 0.3$ \\
Week 4 & $1.2 \pm 0.1$ & $1.2 \pm 0.2$ & $1.1 \pm 0.08$ & $1.2 \pm 0.07$ \\
\hline
\end{tabular}

${ }_{\mathrm{a}, \mathrm{b}}$ Mean values within a row with different superscripts differ $(P<0.05)$.

${ }^{1}$ The data are presented as mean values $\pm \mathrm{SE}$.

blood sampling occurred in the pre-prandial stage and postprandial plasma flavonol concentration in calves decreased rapidly with age (Maciej et al., 2015). The plasma concentrations of the total flavonols were higher $(P<0.05)$ in RU than in CTRL, indicating enrichment of total flavonols in RU, but not in QA. The plasma concentrations of the individual catechins were below the detection limit.

All calves were born spontaneously and were healthy. At birth, BW did not differ among the groups, as planned, and the postnatal growth performance and feed intake were not affected by treatment (Maciej et al., 2015). Interestingly, we found an improved fecal score in CA compared with CTRL calves during the

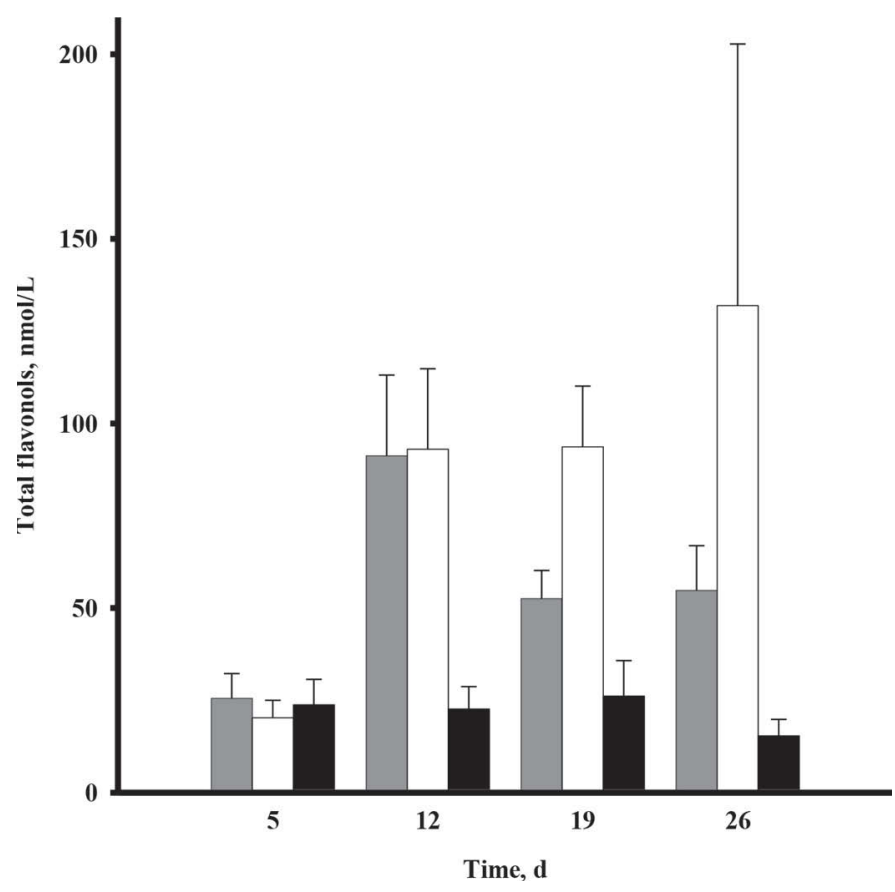

Figure 1. Plasma concentrations of total flavonols from d 5 to 26 after oral administration of $10 \mathrm{mg}$ of quercetin $/ \mathrm{kg}$ of $\mathrm{BW} \times$ day as quercetin aglycone (gray bars) or as rutin (white bars), or no quercetin administration (control group; black bars). Values are least squares means $\pm \mathrm{SE}, \mathrm{n}=7$ per group. first week of life $(P<0.05$; Table 1$)$. In recent studies, GTE were shown to inhibit diarrhea and the number of treatments in newborn calves (Ishihara et al., 2001). Severe diarrhea occurred between d 3 and 20 in all groups, and the fecal score was highest in wk 2 (fecal score 1.62) and wk 3 (fecal score 1.54; Table 1). Each calf had, on average, $2 \mathrm{~d}$ of strong diarrhea, indicated by a fecal score of 4 . In some cases, the diarrhea may have resulted from cryptosporidiosis that was diagnosed when microbes were determined in feces.

The markers for antioxidative capacity (TEAC, FRAP; Figures 2A and 2B) changed over time, with TEAC increasing and FRAP decreasing from $\mathrm{d} 1$ to 5 and then remaining relatively stable in all groups. The measurements of the plasma antioxidant capacity were not influenced by the flavonoid feeding, but the sharp changes in both markers from d 1 to 5 highlight the role of colostrum feeding on postnatal antioxidative status (Blum and Hammon, 2000; Hammon et al., 2013). Measurement of TEAC is dependent on the vitamin $\mathrm{E}$ analog Trolox, and vitamin $\mathrm{E}$ is provided by colostrum feeding (Blum et al., 1997). Our findings on FRAP agree with those of Gaál et al. (2006), which also showed a decline of this parameter after the first colostrum intake. Urea is a major factor influencing FRAP (Benzie and Strain, 1996), and decreasing plasma urea concentrations may contribute to the decremental changes of FRAP in the plasma.

The concentrations of the oxidative stress markers (thiobarbituric acid reactive substances, F2-isoprostanes) revealed huge individual variations within groups and did not show group or time differences (Figures $2 \mathrm{C}$ and $2 \mathrm{D}$ ). These markers did not follow a clear time-dependent trend as seen for TEAC and FRAP. In previous calf studies, the plasma concentrations of oxidative stress markers distinctly increased when the calves were sick or stressed (Ahmed and Hassan, 2007). This suggests that feeding flavonoids to healthy calves, as in the present study, may not change the oxidative stress markers. Additionally, the amount of flavonoid used in this study was based on previous studies in pigs (Lesser et al., 2004; Lühring et al., 2011), and the doses 
may thus not be adequate for colostrum- and milk-fed calves. The determination of other parameters, such as protein damage or the determination of vitamins $\mathrm{E}$ and $\mathrm{C}$ or of enzymatic antioxidants might be more appropriate.

The plasma concentrations of the metabolites showed the typical time changes already known for neonatal calves during the first month of life, such as a sharp increase in plasma total protein concentrations, a slight increase in plasma glucose concentrations, and a decrease in plasma lactate and NEFA concentrations during the first week $(P<0.01$, respectively; Table
2; Hadorn et al., 1997; Nussbaum et al., 2002). The plasma insulin and cortisol concentrations decreased after birth $(P<0.001)$ and then remained low (Table 2$)$. All plasma concentrations measured were comparable among groups except for plasma cortisol, where the decrease after birth differed among groups as reflected by the group $\times$ time interaction $(P<0.001)$. We assumed that feeding quercetin may affect the metabolic status in calves, as some authors postulate effects of flavonoids, for example, on glucose metabolism (Cermak et al., 2004; Shetty et al., 2004). However, we found no such effects caused by flavonoid feeding in this study,
A

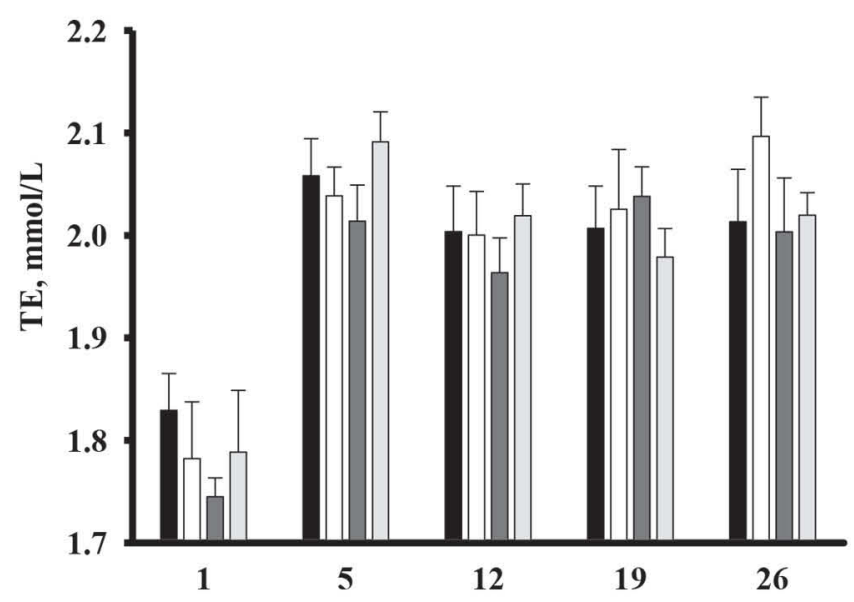

C

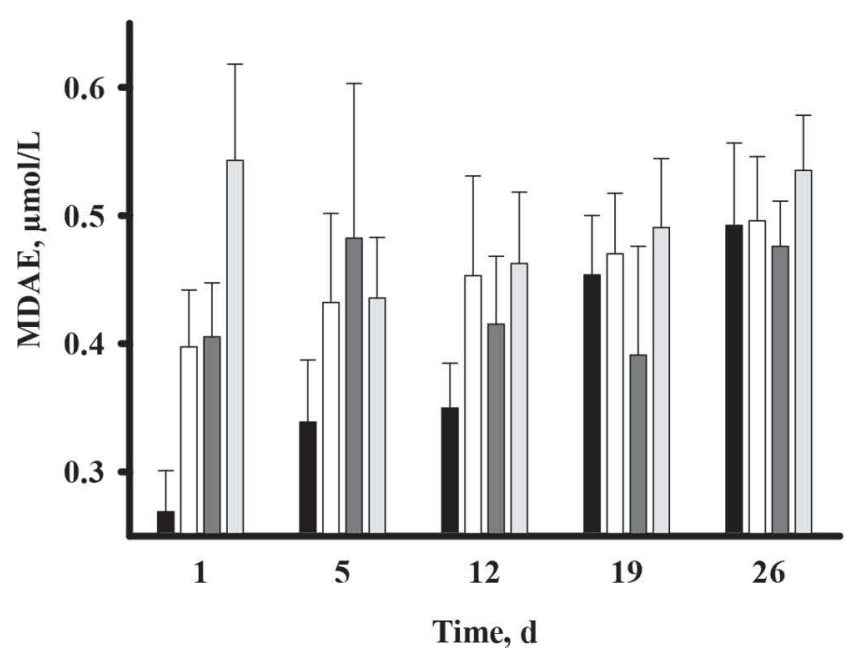

B

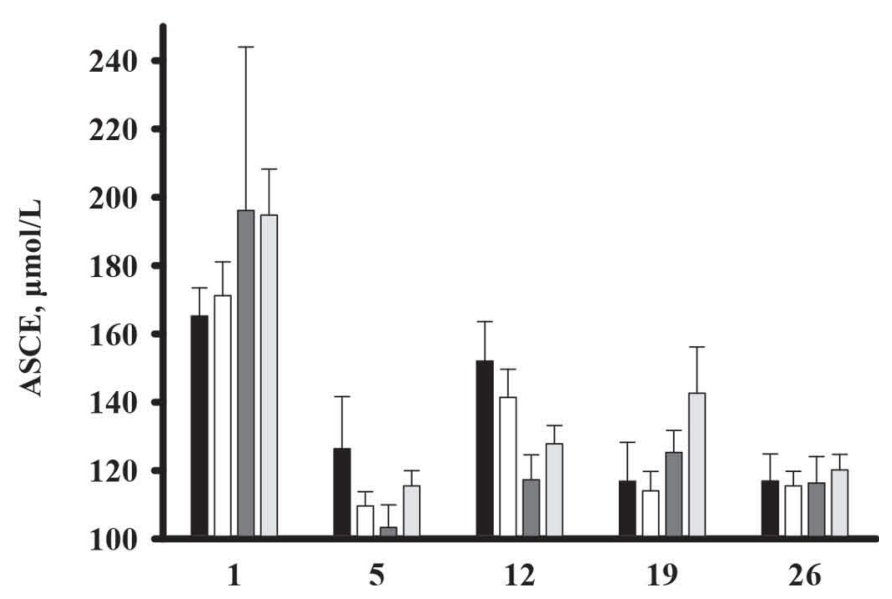

D

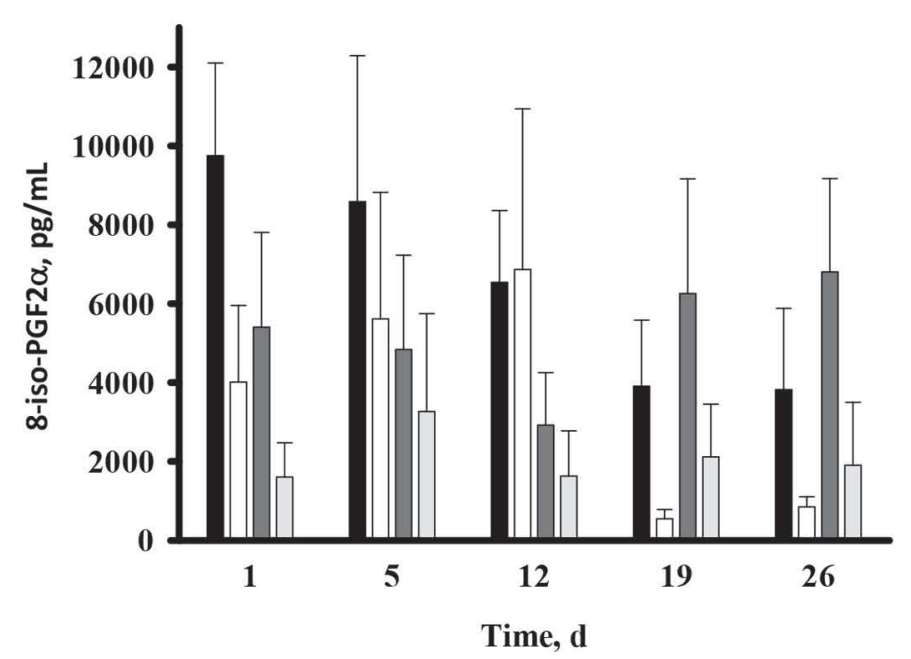

Figure 2. Plasma concentrations of markers for antioxidative capacity $(\mathrm{A}, \mathrm{TEAC}=$ Trolox equivalent antioxidative capacity; $\mathrm{B}, \mathrm{FRAP}=$ ferric reducing ability of plasma) and oxidative stress $(\mathrm{C}$, TBARS = thiobarbituric acid reactive substances; D, F2-isoprostanes; control (black bars), rutin (white bars), quercetin aglycone (dark gray bars), and green tea extract (light gray bars). Values are least squares means \pm SE, $n$ $=7$ per group. $\mathrm{TE}=$ Trolox equivalents; $\mathrm{ASCE}=$ ascorbic acid equivalents; $\mathrm{MDAE}=$ malondialdehyde equivalents; 8 -iso-PGF $\mathrm{P}_{2 \alpha}=8$-isoprostaglandin $\mathrm{F}_{2 \alpha}$. 
Table 2. Blood plasma concentrations of metabolic traits and hormones of calves after feeding no flavonoid (CTRL), quercetin aglycone (QA), rutin $(\mathrm{RU})$, or a green tea extract containing mainly catechins (CA) from d 5 to $26^{1}$

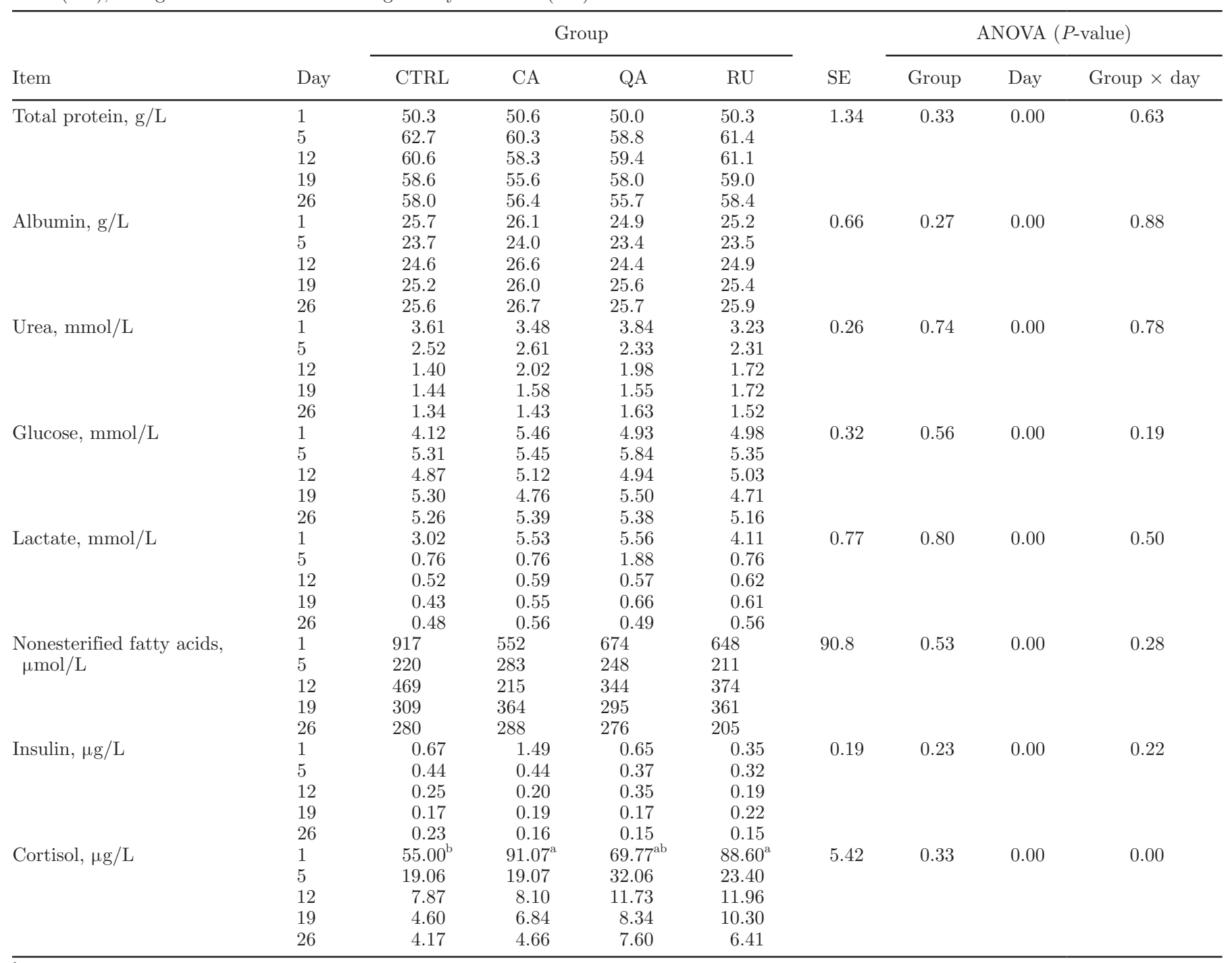

${ }^{1}$ Values are least squares means $\pm \mathrm{SE}, \mathrm{n}=7$ per group. The samples were taken before morning milk feeding.

and any changes simply reflected the physiological changes during the first month of life (Hadorn et al., 1997; Nussbaum et al., 2002; Hammon et al., 2013). The differences in the plasma cortisol concentrations among the groups on $\mathrm{d} 1$ were independent of the flavonoid administration, as the blood was sampled before the flavonoid supplementation.

In conclusion, the effects of the flavonoid treatments were much less than expected. However, the fewer incidences of diarrhea in this study may indicate a potential health-promoting effect of catechins. Feeding higher doses of flavonoids than those used in this study may possibly cause more distinct changes in the metabolic or antioxidative status of neonatal calves.

\section{ACKNOWLEDGMENTS}

We gratefully thank Petra Schulz and Maike Gosse (Christian Albrechts University Kiel) for excellent laboratory assistance as well as Annette Zeyner, Kirsten Büsing (University of Rostock), and Bernd Stabenow (Leibniz Institute for Farm Animal Biology) for providing the experimental facilities. The chicken-egg-derived immunoglobulins (Globigen Life Start 25\%) were generously provided by EW Nutrition GmbH, Visbek, Germany. This work is part of the joint research project Food Chain Plus (FoCus) and was financially supported by the Federal Ministry of Education and Research, Germany (BMFT grant no. 0315538B). 


\section{REFERENCES}

Ahmed, W. M., and S. E. Hassan. 2007. Applied studies on coccidiosis in growing Buffalo-calves with special reference to oxidant/ antioxidant status. World J. Zool. 2:40-48.

Alexandrovich, K. N., and S. E. Antonovna. 2009. Age-dependent level of antioxidant defence system and lipid metabolism state in calves. Int. J. Appl. Res. Vet. Med. 7:73-75.

Benzie, I. F., and J. J. Strain. 1996. The ferric reducing ability of plasma (FRAP) as a measure of "antioxidant power": The FRAP assay. Anal. Biochem. 239:70-76.

Berger, L. M., S. Wein, R. Blank, C. C. Metges, and S. Wolffram. 2012. Bioavailability of the flavonol quercetin in cows after intraruminal application of quercetin aglycone and rutin. J. Dairy Sci. 95:5047-5055.

Blum, J., and H. Hammon. 2000. Colostrum effects on the gastrointestinal tract, and on nutritional, endocrine and metabolic parameters in neonatal calves. Livest. Prod. Sci. 66:151-159.

Blum, J. W., U. Hadorn, H. P. Sallmann, and W. Schuep. 1997. Delaying colostrum intake by one day impairs plasma lipid, essential fatty acid, carotene, retinol and $\alpha$-tocopherol status in neonatal calves. J. Nutr. 127:2024-2029.

Cermak, R., S. Landgraf, and S. Wolffram. 2004. Quercetin glucosides inhibit glucose uptake into brush-border-membrane vesicles of porcine jejunum. Br. J. Nutr. 91:849-855.

di Carlo, G. D., N. Mascolo, A. A. Izzo, and F. Capasso. 1994. Effects of quercetin on the gastrointestinal tract in rats and mice. Phytother. Res. 8:42-45.

Egert, S., J. Tereszczuk, S. Wein, M. J. Müller, J. Frank, G. Rimbach, and S. Wolffram. 2013. Simultaneous ingestion of dietary proteins reduces the bioavailability of galloylated catechins from green tea in humans. Eur. J. Nutr. 52:281-288.

Gaál, T., P. Ribiczeyné-Szabó, K. Stadler, J. Jakus, J. Reiczigel, P. Kövér, M. Mézes, and L. Sümeghy. 2006. Free radicals, lipid peroxidation and the antioxidant system in the blood of cows and newborn calves around calving. Comp. Biochem. Physiol. B Biochem. Mol. Biol. 143:391-396.

Hadorn, U., H. Hammon, R. M. Bruckmaier, and J. W. Blum. 1997. Delaying colostrum intake by one day has important effects on metabolic traits and on gastrointestinal and metabolic hormones in neonatal calves. J. Nutr. 127:2011-2023.

Halliwell, B. 1991. Reactive oxygen species in living systems: Source, biochemistry, and role in human disease. Am. J. Med. 91:14S-22S.

Hammon, H. M., J. Steinhoff-Wagner, J. Flor, U. Schönhusen, and C. C. Metges. 2013. Lactation Biology Symposium: Role of colostrum and colostrum components on glucose metabolism in neonatal calves. J. Anim. Sci. 91:685-695.

Ishihara, N., D.-C. Chu, S. Akachi, and L. R. Juneja. 2001. Improvement of intestinal microflora balance and prevention of digestive and respiratory organ diseases in calves by green tea extracts. Livest. Prod. Sci. 68:217-229.

Kobayashi, Y., M. Miyazawa, A. Kamei, K. Abe, and T. Kojima. 2010. Ameliorative effects of mulberry (Morus alba L.) leaves on hyperlipidemia in rats fed a high-fat diet: Induction of fatty acid oxidation, inhibition of lipogenesis, and suppression of oxidative stress. Biosci. Biotechnol. Biochem. 74:2385-2395.

Larson, L. L., F. G. Owen, J. L. Albright, R. D. Appleman, R. C. Lamb, and L. D. Muller. 1977. Guidelines toward more uniformity in measuring and reporting calf experimental data. J. Dairy Sci. 60:989-991.

Lee, M. J., Z. Y. Wang, H. Li, L. Chen, Y. Sun, S. Gobbo, D. A. Balentine, and C. S. Yang. 1995. Analysis of plasma and urinary tea polyphenols in human subjects. Cancer Epidemiol. Biomarkers Prev. 4:393-399.

Lesser, S., R. Cermak, and S. Wolffram. 2004. Bioavailability of quercetin in pigs is influenced by the dietary fat content. J. Nutr. 134:1508-1511.

Lühring, M., R. Blank, and S. Wolffram. 2011. Vitamin E-sparing and vitamin E-independent antioxidative effects of the flavonol quercetin in growing pigs. Anim. Feed Sci. Technol. 169:199-207.

Maciej, J., C. T. Schäff, E. Kanitz, A. Tuchscherer, R. M. Bruckmaier, S. Wolffram, and H. M. Hammon. 2015. Bioavailability of the flavonol quercetin in neonatal calves after oral administration of quercetin aglycone or rutin. J. Dairy Sci. 98:3906-3917.

Middleton, E., C. Kandaswami, and T. C. Theoharides. 2000. The effects of plant flavonoids on mammalian cells: Implications for inflammation, heart disease, and cancer. Pharmacol. Rev. 52:673751

Naumann, C., and R. Basler. 2004. Die chemische Untersuchung von Futtermitteln. VDLUFA-Verlag, Darmstadt, Germany.

Nussbaum, A., G. Schiessler, H. M. Hammon, and J. W. Blum. 2002. Growth performance and metabolic and endocrine traits in calves pair-fed by bucket or by automate starting in the neonatal period. J. Anim. Sci. 80:1545-1555.

Re, R., N. Pellegrini, A. Proteggente, A. Pannala, M. Yang, and C. Rice-Evans. 1999. Antioxidant activity applying an improved ABTS radical cation decolorization assay. Free Radic. Biol. Med. 26:1231-1237.

Shetty, A. K., R. Rashmi, M. G. R. Rajan, K. Sambaiah, and P. V. Salimath. 2004. Antidiabetic influence of quercetin in streptozotocininduced diabetic rats. Nutr. Res. 24:373-381.

USDA. 2011. Cattle and calves nonpredator death loss in the United States, 2010. USDA-APHIS-VS-CEAH. Fort Collins, CO \#631.1111.

Yagi, K. 1998. Simple assay for the level of total lipid peroxides in serum or plasma. Pages 101-106 in Free Radical and Antioxidant Protocols. D. Armstrong, ed. Humana Press, Buffalo, NY. 